\title{
Impact of intravenous thrombolysis on length of hospital stay in cases of acute ischemic stroke
}

This article was published in the following Dove Press journal:

Neuropsychiatric Disease and Treatment

\author{
Narongrit Kasemsap ${ }^{1,2}$ \\ Nisa Vorasoot ${ }^{1,2}$ \\ Kannikar Kongbunkiat ${ }^{1,2}$ \\ Udomlack Peansukwech' \\ Somsak Tiamkao ${ }^{1,2}$ \\ Kittisak Sawanyawisuth ${ }^{1,3,4}$ \\ 'Department of Medicine, Faculty \\ of Medicine, ${ }^{2}$ North-Eastern Stroke \\ Research Group, ${ }^{3}$ Research Center \\ in Back, Neck, Other Joint Pain and \\ Human Performance (BNOJPH), \\ ${ }^{4}$ Internal Medicine Research Group, \\ Khon Kaen University, Khon \\ Kaen, Thailand
}

Background: There are limited data available on factors associated with length of stay (LOS) in cases of acute ischemic stroke according to Poisson analysis, which is more appropriate than other methods.

Materials and methods: We retrospectively reviewed medical summary charts of patients with acute ischemic stroke in 30 hospitals across northeast Thailand, with the main outcome as LOS. Poisson regression was used to examine factors associated with LOS.

Results: We included 898 patients in the analysis; 460 (51.2\%) were male. The median age (interquartile; IQR) was 58 (67-75) years and the median LOS was 5 (4-7) days. The median National Institute of Health Stroke Scale (NIHSS [IQR]) was 8 (4-13). Results of the analysis showed that, after controlling for age, stroke severity, atrial fibrillation, and thrombolytic use, significant variables associated with LOS were moderate stroke (incidence rate ratio [IRR] 95\% confidence interval $[\mathrm{CI}]=1.15$ [range 1.01-1.30], $P=0.040)$, severe stroke (IRR $[95 \% \mathrm{CI}]=1.27$ [1.09-1.47], $P=0.002$ ), thrombolytic use (IRR [95\% CI] $=0.68[0.60-0.76], P<0.001$ ), and atrial fibrillation (IRR [95\% CI] $=1.15[1.02-1.30], P=0.023$ ). After adjusting for complications, thrombolytic use remained significantly associated with decreased LOS (IRR [95\% CI] $=0.74$ [0.67-0.83], $P=0.001$ ). Other significant factors were atrial fibrillation (IRR $[95 \% \mathrm{CI}]=1.14$ [1.02-1.28], $P=0.018$ ), pneumonia (IRR [95\% CI] $=1.48$ [1.30-1.68], $P<0.001$ ), and urinary tract infection (IRR [95\% CI] $=1.41[1.14-1.74], P=0.001$ ).

Conclusion: According to Poisson analysis, intravenous thrombolysis, atrial fibrillation, pneumonia, and urinary tract infection are associated with LOS in cases of acute ischemic stroke, regardless of age, stroke severity, comorbidities, or complications.

Keywords: acute ischemic stroke, length of stay, thrombolysis

\section{Introduction}

Stroke is the second most common cause of death and disability in Thailand. In 2015, according to the Institute of Health Metrics and Evaluation, there were 1,204.5 years of life lost (YLL) per 100,000 population due to premature mortality from stroke. ${ }^{1}$ The prevalence of stroke in Thailand is estimated to be $1.88 \%$ among adults 45 years old and older. ${ }^{2}$ Expenses incurred from inpatients with stroke may be related to length of stay (LOS). A study from Greece found that the direct cost of acute ischemic stroke admission was $€ 331.9$ per day. ${ }^{3}$ In Thailand, the average hospitalization costs of acute ischemic stroke without thrombolysis use were US\$691 and, with thrombolysis use, US\$1,840. The cost of $50 \mathrm{mg}$ Actilyse $^{\circledR}$ (alteplase; Boehringer Ingelheim, Ingelheim am Rhein, Germany) was US\$629.87. ${ }^{4}$ There are several factors associated with LOS as well as costs of stroke hospitalization such as age of the patient, stroke subtype, atrial fibrillation, stroke severity, and comorbidities..$^{5-7}$
Correspondence: Narongrit Kasemsap Department of Medicine, Faculty of Medicine, Khon Kaen University, I 23 Mitraparp Highway, Khon Kaen 40002, Thailand

Tel +66 43363664

Fax +6643348399

Email naroka@kku.ac.th 
In the era of intravenous thrombolytic treatment (IVT), early or short onset to IVT has decreased the LOS in patients with acute ischemic stroke $\mathrm{e}^{8-10}$ and lowered hospital costs. A national study from Thailand found that the average cost of hospitalization for cases of cerebral infarction with IVT was higher than those without IVT (US\$1,840 vs US\$691). ${ }^{4}$ The higher costs of patients with IVT were due to costs of IVT itself. The cost of $50 \mathrm{mg}$ alteplase in Thailand was already US\$629.87. ${ }^{4}$ Although one study from Taiwan found that IVT reduced LOS by $47 \%$, the multivariate logistic model was built without including stroke complications. ${ }^{11}$ Theoretically, a generalized linear model (GLM) with Poisson regression may be more appropriate to evaluate the association with LOS due to the count data character of LOS. ${ }^{12}$ The previous study showed that GLM with Poisson regression had the greatest consistency in prediction of LOS in patients with coronary artery bypass surgery. This study aimed to examine factors associated with LOS in patients with acute ischemic stroke using the more appropriate GLM model with Poisson regression and a wider array of studied variables.

\section{Materials and methods Study area and population}

This was a retrospective study conducted in northeast Thailand. The study period was from October 2015 to August 2016. The inclusion criteria specified that adult patients admitted with acute ischemic stroke in any level of the hospital in northeast Thailand were eligible. Thirty hospitals participated in this study including primary, secondary, and tertiary/university hospitals. There are four Ministry of Public Health service areas in northeast Thailand. We randomly sampled patients from the list (which contained a total of 1,000 patients from four service areas) and stratified them by level of hospital. We included 40 patients from each tertiary/university hospital and 20 from each primary or secondary hospital, and randomly selected patient who were treated by intravenous thrombolysis and had not received a 1:1 ratio of the case selection process. Exclusion criteria were misdiagnosis of acute ischemic stroke or death after admission. ${ }^{13}$

\section{Data collection and terminology}

Data of all eligible patients were extracted from the admission medical records that were sent to the National Health Security Office (NHSO) for reimbursement purposes. Baseline clinical data, treatment, and outcomes of each patient were recorded. The National Institute of Health Stroke Scale (NIHSS) was classified into three categories: mild (0-6), moderate (7-15), and severe (16-42). LOS was calculated as total length of hospital stay in days from admission until discharge. Ischemic stroke subtype was classified according to the Trial of ORG 10172 in Acute Stroke Treatment (TOAST). ${ }^{14}$ The severity of stroke was classified by the NIHSS. ${ }^{15}$ If there were admission values that were missing from the NIHSS, we estimated them on the basis of the history and physical examinations recorded in the medical records. With regard to complications of acute stroke, an intracerebral hemorrhage after acute ischemic stroke included both asymptomatic and symptomatic manifestations. Those with intracerebral hemorrhage of more than $30 \%$ of the infarcted area with substantial mass effect were defined as the symptomatic group. ${ }^{16}$ The study protocol was approved by the ethics committee on human research, Khon Kaen University (HE 591294). The Khon Kaen University Ethics Committee on human research granted a waiver of informed consent for retrospective chart review study. All data were anonymized and de-identified prior to the analysis that was conducted. This study was registered in the Thai Clinical Trials Registry (TCTR20160901001).

\section{Statistical analysis}

Baseline characteristics were described using mean (standard deviation) or median (interquartile range [IQR]) for continuous data and frequencies for categorical data. All continuous variables were tested for normal distribution and equality of variances. Generalized linear models (GLMs) were used for examining the association of variables with LOS in unadjusted outcomes (Model A). A GLM was used to determine variables that were independently associated with length of hospital stay. A GLM was used with the Poisson family and $\log$ for link functions. A Poisson regression was used because LOS is used to count data and correct for overdispersion of that model using the Bootstrap test. LOS outcomes were adjusted for age, severity of stroke, thrombolysis use, and presence of atrial fibrillation (Model B). Model C included variables in Model B as well as complications of stroke, including pneumonia, urinary tract infection, sepsis, acute kidney injury, gastrointestinal bleeding, and any intracerebral hemorrhage. The discrimination of the model was assessed using the Akaike Information Criterion (AIC) and Bayesian Information Criterion (BIC) as a measure of fit. All statistical analyses were conducted with STATA 10.1 (StataCorp, College Station, TX, USA).

\section{Results}

There were 6,655 patients with acute ischemic stroke admitted in the four service areas. A total of 1,000 patients 
(15.03\%) were eligible for inclusion in this study. Of those, 47 patients were excluded due to misdiagnosis of stroke, and 55 due to death following admission. Causes of death were pneumonia $(n=29)$, brain herniation $(n=7)$, sepsis $(n=6)$, gastrointestinal bleeding $(n=4)$, intracerebral hemorrhage $(n=3)$, respiratory failure $(n=2)$, cardiac arrest $(n=2)$, pulmonary embolism $(n=1)$, and myocardial infarction $(n=1)$. Of the remaining 898 patients, 167 (18.60\%), 597 (66.48\%), and 134 (14.89\%) were from primary, secondary, and tertiary/ university hospitals, respectively. With regard to characteristic data (Table 1), sex distribution was almost equal to 1 , and the median age and NIHSS were 58 years and 8 , respectively. The most common stroke subtype was largeartery atherosclerosis (309 patients; 34.41\%), hypertension was the comorbidity with the highest proportion (48.6\%), and pneumonia was the most commonly found complication after acute ischemic stroke $(21.3 \%)$. Thrombolytic therapy

Table I Clinical characteristics of patients with acute ischemic stroke admitted at the primary, secondary, and tertiary/university hospitals throughout northeast Thailand

\begin{tabular}{|c|c|}
\hline Characteristics & $n=898$ \\
\hline Male & $460(51.22)$ \\
\hline Median (first-third IQR) age, years & $58(67-75)$ \\
\hline Median (first-third IQR) NIHSS & $8(4-13)$ \\
\hline Mild (0-6) & $362(40.31)$ \\
\hline Moderate $(7-15)$ & $386(42.98)$ \\
\hline Severe $(16-42)$ & $150(16.70)$ \\
\hline \multicolumn{2}{|l|}{ Stroke classification } \\
\hline Large-artery atherosclerosis & $309(34.41)$ \\
\hline Cardioembolism & $236(26.28)$ \\
\hline Small-vessel occlusion & $339(37.75)$ \\
\hline Other etiology & $2(0.22)$ \\
\hline Undetermined etiology & $8(0.89)$ \\
\hline \multicolumn{2}{|l|}{ Comorbidities } \\
\hline Hypertension & $436(48.55)$ \\
\hline Diabetes mellitus & $229(25.50)$ \\
\hline Dyslipidemia & $235(26.17)$ \\
\hline Atrial fibrillation & $218(24.28)$ \\
\hline Coronary artery disease & $47(5.23)$ \\
\hline Previous stroke & II $5(12.8 I)$ \\
\hline Chronic kidney disease & $40(4.45)$ \\
\hline Thrombolytic use & 422 (46.99) \\
\hline \multicolumn{2}{|l|}{ Complications } \\
\hline Pneumonia & $191(21.27)$ \\
\hline Urinary tract infection & $45(5.01)$ \\
\hline Sepsis & $40(4.45)$ \\
\hline Gastrointestinal bleeding & $19(2.12)$ \\
\hline Acute kidney injury & $35(3.90)$ \\
\hline Heart failure & $20(2.23)$ \\
\hline Brain herniation & $38(4.23)$ \\
\hline Intracerebral hemorrhage & $69(7.68)$ \\
\hline
\end{tabular}

Note: Data presented as number (percentage), unless indicated otherwise.

Abbreviations: IQR, interquartile range; NIHSS, National Institute of Health Stroke Scale. was prescribed in 422 cases $(46.99 \%)$. The overall median LOS was 5 (range 4-7) days. There were no differences in length of hospital stay among hospital levels; primary or community hospital 5 days (IQR 4-6), secondary hospital 5 days (IQR 4-7), and tertiary hospital 5 days (IQR 4-8), with $P$-value 0.19 .

There were nine factors associated with LOS according to univariate analysis including age, stroke severity, thrombolysis use, atrial fibrillation, pneumonia, urinary tract infection, sepsis, gastrointestinal bleeding, and acute kidney injury (Model A; Table 2). After adjustment using Model B (Table 2), age was no longer significant. In addition, age and stroke severity were not significant according to Model $\mathrm{C}$ (Table 2). There were four independent factors according to Model $\mathrm{C}$ including thrombolytic therapy, atrial fibrillation, pneumonia, and urinary tract infection. Pneumonia had the highest incidence rate ratio (IRR) at 1.48 (1.30-1.68), whereas thrombolytic therapy was negatively associated with LOS by 0.74 (0.67-0.83). Additionally, Model C had better overall fit of model than Model B according to AIC and BIC statistics.

\section{Discussion}

Using Poisson regression analysis for LOS prediction in acute ischemic stroke, we found four independent factors associated with LOS including thrombolysis therapy, atrial fibrillation, pneumonia, and urinary tract infection (Table 2). These predictors were significantly related to LOS regardless of age, stroke severity, stroke subtype, comorbidities, or complications (Model C). Note that the IVT rate in this study may be higher than in real-life clinical practice or than previously reported in other countries due to the study design. ${ }^{17-19} \mathrm{We}$ randomly selected patients with acute ischemic stroke who received the IVT or not equally. Therefore, the results of this study are applicable if the rate of IVT is nearly 50\%.

Compared with the logistic regression model to predict LOS in acute ischemic stroke treatment, ${ }^{15}$ only thrombolytic therapy significantly shortened hospital stay (adjusted odds ratio 0.49 ; IRR 0.74 in this study). However, age, stroke severity, and diabetes were not significant factors associated with LOS according to this Poisson analysis (Table 2). However, the logistic model found that these three factors increased LOS by more than 7 days (up to 19.15 times). ${ }^{15}$ These differences may be due to differences in the statistical analytical methods used and the factors included in the model. In Model C, complications were also included.

In previous studies, atrial fibrillation, diabetes, hyperglycemia, and ischemic stroke subtype have been found to be correlated with increased LOS. ${ }^{5,20,21}$ In this study, we found that only atrial fibrillation was strongly associated with LOS 
Table 2 Predictors for length of stay in patients with acute ischemic stroke according to three different models

\begin{tabular}{|c|c|c|c|c|c|c|}
\hline \multirow[t]{2}{*}{ Characteristics } & \multicolumn{2}{|l|}{ Model A* } & \multicolumn{2}{|l|}{ Model B\# } & \multicolumn{2}{|l|}{ Model C } \\
\hline & IRR (95\% CI) & $P$-value & IRR (95\% CI) & $P$-value & IRR (95\% CI) & $P$-value \\
\hline Age & $1.00(1.00-1.01)$ & 0.005 & $1.00(0.99-1.00)$ & 0.763 & $1.00(0.99-1.00)$ & 0.638 \\
\hline \multicolumn{7}{|l|}{ Stroke severity } \\
\hline Mild (0-6) & I & & I & & I & \\
\hline Moderate (7-15) & $1.02(0.96-1.08)$ & 0.552 & $1.15(1.01-1.30)$ & 0.040 & $1.05(0.94-1.78)$ & 0.389 \\
\hline Severe $(16-42)$ & $1.10(1.02-1.19)$ & 0.012 & $1.27(1.09-1.47)$ & 0.002 & $1.00(0.87-1.15)$ & 0.962 \\
\hline Thrombolytic therapy & $0.68(0.68-0.76)$ & $<0.001$ & $0.68(0.60-0.76)$ & $<0.001$ & $0.74(0.67-0.83)$ & $<0.00$ I \\
\hline \multicolumn{7}{|l|}{ Risk factors } \\
\hline Atrial fibrillation & $1.19(1.12-1.26)$ & $<0.001$ & $1.15(1.02-1.30)$ & 0.023 & $1.14(1.02-1.28)$ & 0.018 \\
\hline \multicolumn{7}{|l|}{ Complications } \\
\hline Pneumonia & $1.66(1.57-1.76)$ & $<0.001$ & - & - & $1.48(1.30-1.68)$ & $<0.00 \mathrm{I}$ \\
\hline Urinary tract infection & $1.74(1.58-1.91)$ & $<0.001$ & - & - & I.4I (I.I4-I.74) & 0.001 \\
\hline Sepsis & $1.80(1.63-1.99)$ & $<0.001$ & - & - & $1.42(0.95-2.12)$ & 0.085 \\
\hline Gastrointestinal bleeding & $1.53(1.32-0.178)$ & $<0.001$ & - & - & $1.04(0.65-1.65)$ & 0.880 \\
\hline Acute kidney injury & $1.46(1.31-1.64)$ & $<0.001$ & - & - & $1.09(0.86-1.38)$ & 0.465 \\
\hline Intracerebral hemorrhage & $0.98(0.89-1.08)$ & 0.690 & - & - & $\mathrm{I} .0 \mathrm{I}(0.84-1.2 \mathrm{I})$ & 0.933 \\
\hline
\end{tabular}

Notes: Poisson regression analysis regressing length of stay on the independent variable. Incidence rate ratios (IRRs) and their corresponding $95 \%$ confidence intervals (Cls) are depicted. *Unadjusted. \#Adjusted for age, stroke severity, and atrial fibrillation. ${ }^{\ddagger}$ Adjusted for age, stroke severity, atrial fibrillation, pneumonia, urinary tract infection, sepsis, gastrointestinal bleeding, acute kidney injury, and intracerebral hemorrhage.

according to all three of the models used (Table 2). The presence of atrial fibrillation increased LOS by $14 \%-19 \%$ according to these three models. Patients with acute ischemic stroke with atrial fibrillation tend to have more severe strokes and, subsequently, more complications. Additionally, the mortality rate in cases of acute stroke with atrial fibrillation was significantly higher than in those without atrial fibrillation (14.1\% vs $6.2 \%){ }^{4,21}$ Furthermore, atrial fibrillation was found to increase hospitalization expense. ${ }^{22-24}$

Among the four complications included in Model C, only pneumonia and urinary tract infection were independent predictors (Table 2). As previously reported, pneumonia is the most common medical complication after acute ischemic stroke and is associated with the development of other complications such as urinary tract infection, gastrointestinal bleeding, and decubitus ulcer. ${ }^{25}$ These complications have an impact on LOS and are independently associated with poor functional outcomes. ${ }^{26-28}$ Moreover, these complications have been found to increase mortality. ${ }^{29}$

In our study, LOS was shorter than in previously published studies. ${ }^{11,30,31}$ The mean/median of LOS in acute ischemic stroke settings in Taiwan, Singapore, and Germany were 7, 9 , and 7 days, respectively. In Thailand, the patient with acute ischemic stroke was transferred from the tertiary or secondary hospital to a local hospital or discharged home after stroke stabilization. After discharge, further home rehabilitations are undertaken by village health volunteers and nurses.

The LOS is the most important contributor to hospital cost in patients with acute ischemic stroke., ${ }^{3,53}$ Although IVT increased the direct medical cost of treatment, it significantly reduced LOS. This may decrease hospital expenditure and significantly reduce the functional dependence of patients in the long term. Pharmacoeconomics studies have found that IVT is an extremely cost-effective treatment for patients with acute ischemic stroke in both developed and developing countries. ${ }^{33,34}$ Doctors, therefore, should be encouraged to increase their usage of IVT.

\section{Strengths and limitations}

This study had several strengths. First, it was conducted with hospitals of various levels, including primary, secondary, and tertiary/university hospitals, across northeast Thailand, indicating that the findings can generalized to the Thai population as a whole. Second, our analysis included all factors that could affect LOS including stroke severity, risk factors, and complications. Third, our analytical models can be used to predict the LOS of patients with acute ischemic stroke at admission (Model B) and during in-hospital stay (Model C).

There were some limitations as well. First, LOS in this study included only LOS of acute stroke treatment; therefore, a direct comparison cannot be made with studies that included the post-treatment rehabilitation period. Second, due to the retrospective nature of study, some factors may be missing (in particular, initial NIHSS). In order to compensate for this, we used data from medical records to estimate NIHSS. Third, the severity of stroke or atrial fibrillation may prolong LOS or cause post-stroke complications. However, this was solved by using multivariate analysis. Finally, thrombectomy is not widely available in Thailand. None of the patients in this study underwent thrombectomy. 


\section{Conclusion}

Intravenous thrombolysis, atrial fibrillation, pneumonia, and urinary tract infection were found to be associated with LOS in cases of acute ischemic stroke regardless of age, stroke severity, comorbidities, or complications according to Poisson analysis.

\section{Acknowledgments}

The authors would like to thank Dylan Southard via the Publication Clinic at Khon Kaen University (Thailand) for proofreading this article. This work was supported by the Thailand Research Fund (TRF) and National Health Security Office (NHSO). This research was supported by the Faculty of Medicine, Khon Kaen University (grant no IN59314).

\section{Disclosure}

The authors report no conflicts of interest in this work.

\section{References}

1. Institute of Health Metric and Evaluation. Available from: http://www. healthdata.org/thailand. Accessed February 6, 2017.

2. Suwanwela NC. Stroke epidemiology in Thailand. J Stroke. 2014; 16(1):1-7.

3. Gioldasis G, Talelli P, Chroni E, Daouli J, Papapetropoulos T, Ellul J. In-hospital direct cost of acute ischemic and hemorrhagic stroke in Greece. Acta Neurol Scand. 2008;118(4):268-274.

4. Kongbunkiat K, Kasemsap N, Thepsuthammarat K, Tiamkao S, Sawanyawisuth K. National data on stroke outcomes in Thailand. J Clin Neurosci. 2015;22(3):493-497.

5. Huang YC, Hu CJ, Lee TH, et al. The impact factors on the cost and length of stay among acute ischemic stroke. J Stroke Cerebrovasc Dis. 2013;22(7):E152-E158.

6. Wang G, Zhang Z, Ayala C, Dunet DO, Fang J, George MG. Costs of hospitalization for stroke patients aged 18-64 years in the United States. J Stroke Cerebrovasc Dis. 2014;23(5):861-868.

7. Wang G, Joo H, Tong X, George MG. Hospital costs associated with atrial fibrillation for patients with ischemic stroke aged 18-64 years in the United States. Stroke. 2016;46(5):1314-1320.

8. Saver JL, Fonarow GC, Smith EE, et al. Time to treatment with intravenous tissue plasminogen activator and outcome from acute ischemic stroke. JAMA. 2013;309(23):2480-2488.

9. Fonarow GC, Zhao X, Smith EE, et al. Door-to-needle times for tissue plasminogen activator administration and clinical outcomes in acute ischemic stroke before and after a quality improvement initiative. JAMA. 2014;311(16):1632-1640.

10. Ido MS, Okosun IS, Bayakly R, et al. Door to intravenous tissue plasminogen activator time and hospital length of stay in acute ischemic stroke patients, Georgia, 2007-2013. J Stroke Cerebrovasc Dis. 2016; 25(4):866-871.

11. Hung LC, Hu YH, Sung SF. Exploring the impact of intravenous thrombolysis on length of stay for acute ischemic stroke: a retrospective cohort study. BMC Health Serv Res. 2015;15(1):404.

12. Austin PC, Rothwell DM, Tu JV. A comparison of statistical modeling strategies for analyzing length of stay after CABG surgery. Health Serv Outcomes Res Methodol. 2002;3:107-133.

13. Kang JH, Bae HJ, Choi YA, Lee SH, Shin HI. Length of hospital stay after stroke: a Korean nationwide study. Ann Rehabil Med. 2016; 40(4):675-681.
14. Adams HP Jr, Bendixen BH, Kappelle LJ, et al. Classification of subtype of acute ischemic stroke. Definitions for use in a multicenter clinical trial. TOAST. Trial of Org 10172 in acute stroke treatment. Stroke. 1993; 24(1):35-41.

15. Schlegel D, Kolb SJ, Luciano JM, et al. Utility of the NIH stroke scale as a predictor of hospital disposition. Stroke. 2003;34(1):134-137.

16. Hacke W, Kaste M, Fieschi C, et al. Randomised double-blind placebocontrolled trial of thrombolytic therapy with intravenous alteplase in acute ischaemic stroke (ECASS II). Second European-Australasian acute stroke study investigators. Lancet. 1998;352(9136):1245-1251.

17. Liu Q, Ranta AA, Abernethy G, Barber PA. Trends in New Zealand stroke thrombolysis treatment rates. NZ Med J. 2017;130(1453):50-56.

18. Dong Q, Dong Y, Liu L, et al. The Chinese Stroke Association scientific statement: intravenous thrombolysis in acute ischaemic stroke. Stroke Vasc Neurol. 2017;2(3):147-159.

19. Kawano H, Levi C, Inatomi $Y$, et al. International benchmarking for acute thrombolytic therapy implementation in Australia and Japan. J Clin Neurosci. 2016;29:87-91.

20. Gofir A, Mulyono B, Sutarni S. Hyperglycemia as a prognosis predictor of length of stay and functional outcomes in patients with acute ischemic stroke. Int J Neurosci. 2017;127(10):923-929.

21. Li Y, Liu H, Wang J, et al. Variable lengths of stay among ischemic stroke subtypes in Chinese general teaching hospitals. PLoS One. 2012; 7(9):e45101.

22. Chu CH, Weng WC, Su FC, et al. Association between atrial fibrillation and three-year mortality in nondiabetic patients with acute first-ever ischemic stroke. J Stroke Cerebrovasc Dis. 2016;25(11):2660-2667.

23. Kongbunkiat K, Kasemsap N, Travanichakul S, Thepsuthammarat K, Tiamkao S, Sawanyawisuth K. Hospital mortality from atrial fibrillation associated with ischemic stroke: a national data report. Int J Neurosci. 2015;125(12):924-928.

24. Li J, Luo W. Hospitalization expenses of acute ischemic stroke patients with atrial fibrillation relative to those with normal sinus rhythm. J Med Econ. 2017;20(2):114-120.

25. Ji R, Wang D, Shen H, et al; China National Stroke Registry (CNSR) Investigators. Interrelationship among common medical complications after acute stroke: pneumonia plays an important role. Stroke. 2013; 44(12):3436-3444.

26. Vermeij FH, Scholte op Reimer WJ, de Man P, et al; Netherlands Stroke Survey Investigators. Stroke-associated infection is an independent risk factor for poor outcome after acute ischemic stroke: data from the Netherlands Stroke Survey. Cerebrovasc Dis. 2009;27(5):465-471.

27. Finlayson O, Kapral M, Hall R, Asllani E, Selchen D, Saposnik G; Canadian Stroke Network; Stroke Outcome Research Canada (SORCan) Working Group. Risk factors, inpatient care, and outcomes of pneumonia after ischemic stroke. Neurology. 2011;77(14):1338-1345.

28. Arboix A, Massons J, García-eroles L, Targa C, Comes E. Clinical predictors of prolonged hospital stay after acute stroke: relevance of medical complications. Int J Clin Med. 2012;3(6):502-507.

29. Ingeman A, Andersen G, Hundborg HH, Svendsen ML, Johnsen SP. In-hospital medical complications, length of stay, and mortality among stroke unit patients. Stroke. 2011;42(11):3214-3218.

30. Ng YS, Tan KH, Chen C, Senolos GC, Chew E, Koh GC. Predictors of acute, rehabilitation and total length of stay in acute stroke: a prospective cohort study. Ann Acad Med Singapore. 2016;45(9):394-403.

31. Hillmann S, Wiedmann S, Rücker V, et al; German Stroke Register Study Group (ADSR). Stroke unit care in germany: the german stroke registers study group (ADSR). BMC Neurol. 2017;17(1):49.

32. Buisman LR, Tan SS, Nederkoorn PJ, Koudstaal PJ, Redekop WK. Hospital costs of ischemic stroke and TIA in the Netherlands. Neurology. 2015;84(22):2208-2215.

33. Tan Tanny SP, Busija L, Liew D, Teo S, Davis SM, Yan B. Cost-effectiveness of thrombolysis within 4.5 hours of acute ischemic stroke: experience from Australian stroke center. Stroke. 2013;44(8):2269-2274.

34. Pan Y, Chen Q, Zhao X, et al; TIMS-CHINA Investigators. Costeffectiveness of thrombolysis within 4.5 hours of acute ischemic stroke in China. PLoS One. 2014;9(10):e110525. 


\section{Publish your work in this journal}

Neuropsychiatric Disease and Treatment is an international, peerreviewed journal of clinical therapeutics and pharmacology focusing on concise rapid reporting of clinical or pre-clinical studies on a range of neuropsychiatric and neurological disorders. This journal is indexed on PubMed Central, the 'PsycINFO' database and CAS,

and is the official journal of The International Neuropsychiatric Association (INA). The manuscript management system is completely online and includes a very quick and fair peer-review system, which is all easy to use. Visit http://www.dovepress.com/testimonials.php to read real quotes from published authors.

Submit your manuscript here: http://www.dovepress.com/neuropsychiatric-disease-and-treatment-journal 